\title{
Combined Supra- and Infra-Tentorial Retro-Labyrinthine Pre-sigmoid Approach
}

\author{
Nick Thomas $^{1} \cdot$ Eleni Maratos $^{1} \cdot$ Sinan Barazi $^{1}$
}

Published online: 17 May 2019

(C) The Author(s) 2019

\begin{abstract}
Purpose of Review This chapter describes the background, indications and key aspects of the combined supra- and infra-tentorial retro-labyrinthine pre-sigmoid approach. This approach is most commonly used for petrous apex meningiomata and vascular pathologies of the mid and upper basilar trunk.

Recent Findings Our pre-operative evaluation includes MRI, CT to assess pneumatisation of the petrous bone and vascular imaging where necessary. Audiology and ophthalmology baseline assessments are performed if appropriate.

Summary The nuances of the operative technique are discussed with an emphasis on avoidance of complications.
\end{abstract}

Keywords Supra-and infra-tentorial $\cdot$ Retro-labyrinthine pre-sigmoid approach

\section{Background}

Access to pathologies at the petrous apex and the rostral brainstem is surgically demanding. There are a number of possible approaches including the retrosigmoid craniotomy, the orbito-zygomatic craniotomy, the anterior and lateral petrosectomy and other trans-petrous approaches. The focus of this chapter is the combined supra- and infra-tentorial retrolabyrinthine pre-sigmoid approach. By removing the petrous bone and cutting the tentorium, this approach allows for the creation of a large corridor of access to expose the anterolateral brainstem, petrous apex and upper and mid basilar trunk and its branches and provides access both above and below the tentorium. This follows the skull base concept of creating space by removing the bone and therefore minimising brain retraction. Other approaches do not create such exposure.

This approach has a number of advantages. For example, it obviates the need for the Sylvian fissure split that is required with the orbito-zygomatic approach. The access also enables

This article is part of the Topical Collection on OTOLOGY: Tumors of the Ear and Lateral Skull Base

Nick Thomas

nick.thomas1@nhs.net

1 Department of Neurosurgery, King's College Hospital, London SE5 9RS, UK early identification of cranial nerves that are displaced laterally by a tumour (particularly the third, fourth and fifth).

\section{Introduction}

In the early 1960 s, the operating microscope was introduced to neurosurgery. The superior illumination, optics and magnification enabled dissection of the brain and vasculature to be performed more safely at depth with improved haemostasis, ultimately leading to reduced morbidity and mortality.

Pertinent to skull base approaches, House and Hitselberger refined the anatomical definition of the temporal bone and popularised the trans-labyrinthine approach to vestibular schwannoma and reported on combining this approach with the sub-occipital access[1•].

In the early 1970s, King and Morrison [2•] at the London Hospital described extending a standard trans-labyrinthine approach with ligation of the superior petrosal sinus enabling the surgical field to be widened.

Hakuba noted that division of sigmoid sinus employed to increase exposure occasionally resulted in venous infarction. He developed the anterior petrosectomy approach dividing the superior petrosal sinus.

Spetzler [3•*] reported on 46 petroclival lesions where an anterior petrosectomy was performed either trans-labyrinthine or retro-labyrinthine, combining this with a temporal 
craniotomy. Further refinements of the approach have been reported by Fukushima.

\section{Indications}

This approach is suitable for petrous apex lesions such as meningiomata, intrinsic antero-lateral brainstem lesions such as cavernomas and upper/mid basilar artery trunk aneurysms and vascular anomalies.

\section{Pre-operative Evaluation}

All tumour cases are discussed in a multi-disciplinary combined neurosurgical and otolaryngological meeting. Similarly, vascular cases are discussed in a multi-disciplinary setting.

In the skull base tumour clinic, a full history and examination is performed with a particular attention paid to cranial nerve function. Informed consent is obtained after the risks are explained. Due to the risk of a post-operative seizure, the driving vehicle licence authority in the UK will revoke the patient's licence for 1 year.
The pre-operative work-up includes pre- and post-contrast MR imaging for all lesions and angiography for vascular lesions. The relationship of the cranial nerves to the tumour should be identified if possible. The dominance of the transverse and sigmoid sinuses and the position of the draining veins particularly the vein of Labbé should be identified.

CT imaging is appropriate to identify the size and extent of pneumatisation of the petrous bone which will indicate the ease of surgery and the extent of access. It will also identify any involvement of the bone with invasive tumours.

Pre-operative speech discrimination audiology should be performed. Hearing loss may be a presenting complaint. Not only is it important to document the hearing loss on the side to be operated upon but also to ensure that there is normal/ adequate hearing on the contralateral side (Fig. 1).

Ophthalmological function may need documenting with respect to ophthalmoparesis and visual acuity.

\section{Pre-operative Management}

If there is evidence of obstructive hydrocephalus associated with a petrous apex tumour, then ventriculo-peritoneal shunting is
Fig. 1 A 38-year-old with mild headache and deafness in the right ear for 1 year and large petrous apex meningioma
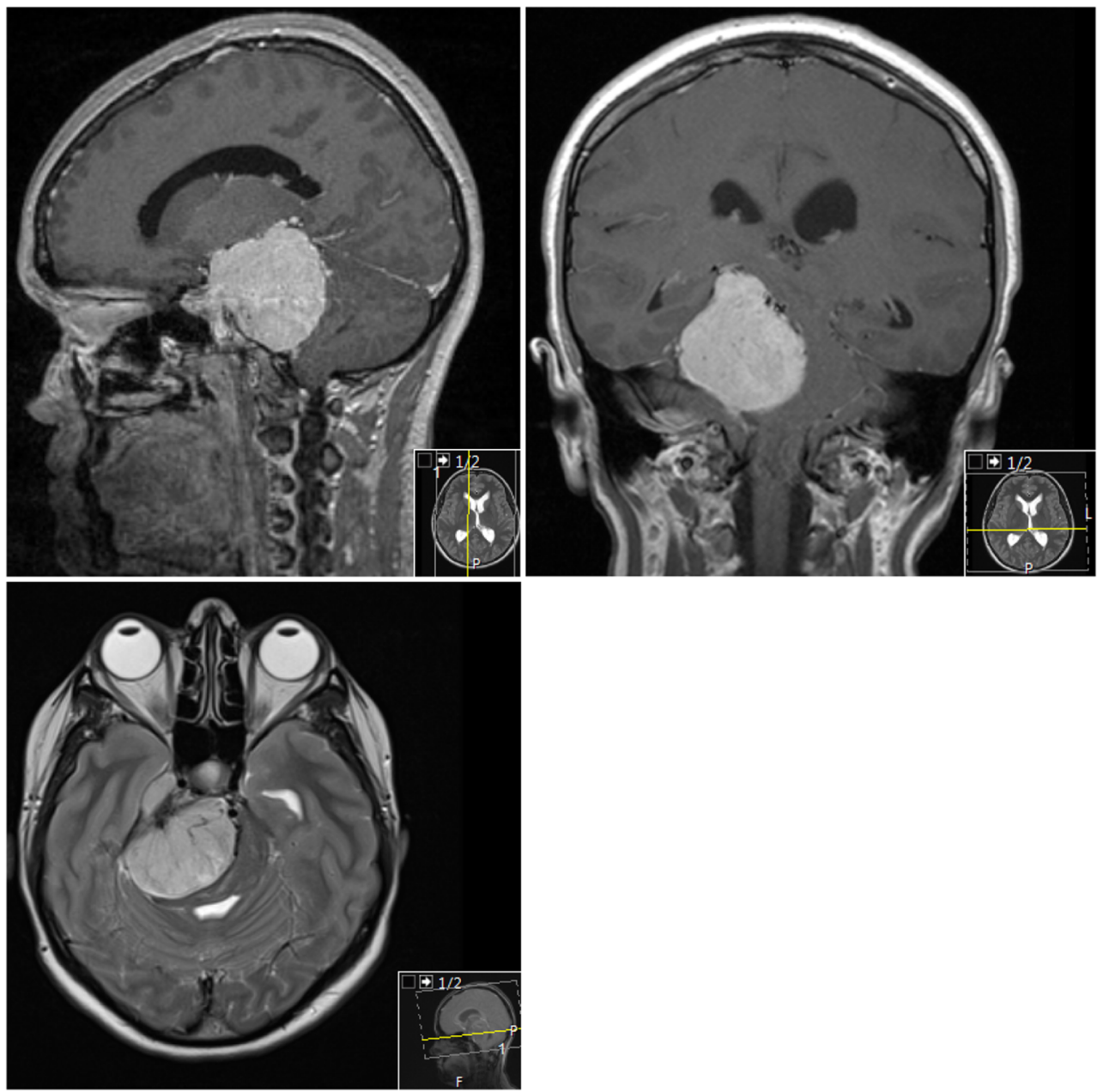
performed a week before definitive tumour resection. We would advocate placing the shunt on the contralateral side to the tumour to avoid it interfering with the second stage surgical incision.

All patients with skull base meningiomata are pre-treated with 5 days of dexamethasone $8 \mathrm{mg}$ once daily with proton pump inhibitor cover prior to admission. There is no evidence for the use of prophylactic anti-convulsants in this setting, and a number of years ago, we witnessed complications related to anti-convulsants including blood dyscrasias and DRESS syndrome. We therefore avoid prophylactic anti-convulsants in this setting.

\section{Operative Technique}

The procedure is carried out under total intravenous anaesthesia (TIVA) with appropriate cardiovascular, respiratory, bispectral index (BIS) and neuro-monitoring including cranial nerve, somatosensory-evoked and motor-evoked potentials (Fig. 2). The TIVA obviates any concerns of interactions between the anaesthetic and the neuro-monitoring. Urinary catheter insertion is required.

\section{Positioning}

The patient is positioned supine in a 3-pin Mayfield head clamp, reverse Trendelenberg with $30^{\circ}$ head-up and rotated contralaterally approximately $80^{\circ}$.

As cerebrospinal fluid can be drained from the basal cisterns and the cisterna magna early after dural opening, we do not routinely use a lumbar drain. Furthermore, we have learned that the mastoid cavity repair required to avoid CSF

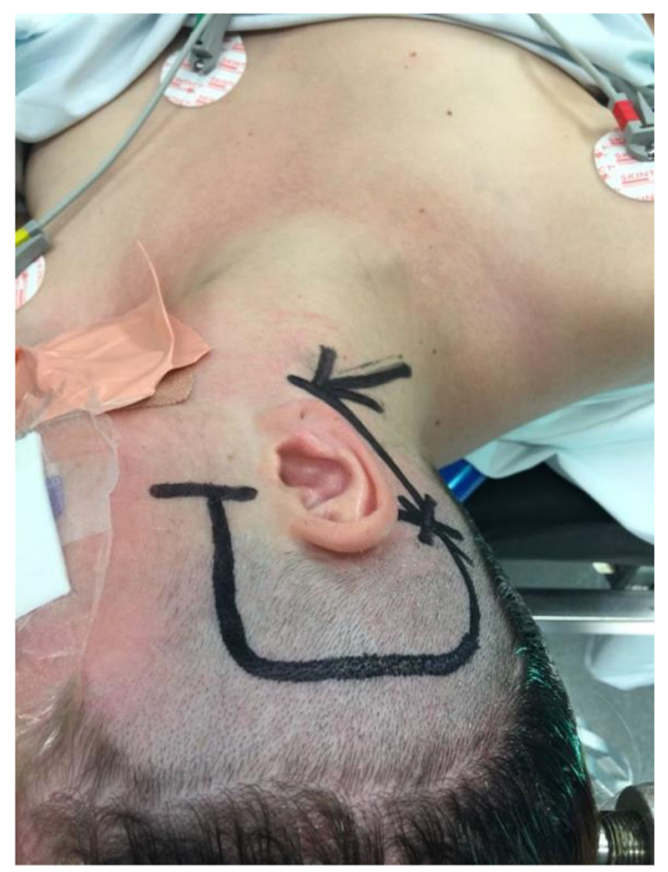

Fig. 2 Positioning and skin incision rhinorrhoea post-operatively does not need to be protected with a lumbar drain.

We do not routinely use image guidance for these procedures but it can be helpful in identifying certain anatomical landmarks.

\section{Skin Incision}

A large C-shaped skin incision is performed extending from the temporal region posteriorly around the pinna to the retroauricular region and down to just below the mastoid tip.

The skin and sub-cutaneous tissues are dissected to expose the temporalis muscle and fascia.

The temporalis fascia and muscle are then elevated together with the pedicle based anteriorly. A small cuff of tissue should be left peripherally so as to be able to re-suture the muscle in its appropriate position at the end of the procedure.

Sternocleidomastoid and the sub-occipital musculature are elevated along with the skin and sub-cutaneous tissue to expose the mastoid to its tip. The muscles do not need to be disarticulated separately.

Once the flap is reflected forward and secured, the root of the zygoma is identified. A line from this to the external occipital protuberance identifies the horizontal level of the transverse sinus. The posterior limit of the junction of the transverse and sigmoid sinus is approximately $1 \mathrm{~cm}$ anterior to the asterion.

A temporal craniotomy is performed which is approximately $5 \times 5 \mathrm{~cm}$ with the antero-inferior extent being at the root of the zygoma and the posterior limit at the junction of the two sinuses. Any inferior lip of temporal bone should be drilled so that the craniotomy is flush with the temporal tegmen.

The middle fossa dura is then elevated to identify the relevant anatomical landmarks including the posterior edge of the mandibular branch of the trigeminal nerve (V3), the middle

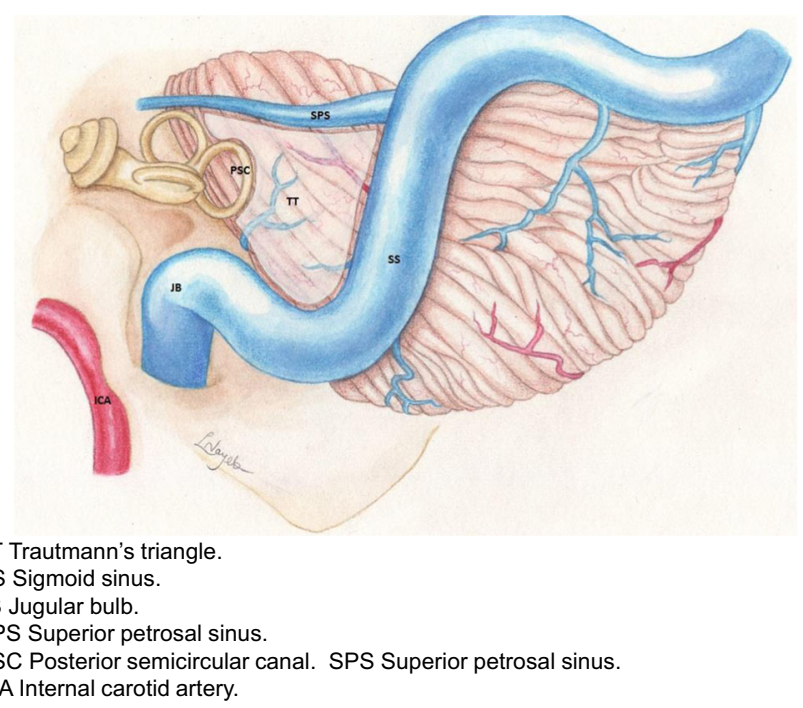

Fig. 3 Trautmann's triangle 
SC Superior semicircular canal LC Lateral semicircular canal PC Posterior semicircular canal SCA Superior cerebellar artery

Fig. 4 Exposure of surgical field

meningeal artery within foramen spinosum, the greater superficial petrosal nerve and the arcuate eminence, often representing the superior semi-circular canal.

Dissection of the petrous bone proceeds with a mastoidectomy to identify the facial nerve within the fallopian canal which is skeletonised along with the sigmoid sinus and jugular bulb in order to maximise bone resection and operative exposure. The labyrinthine block is outlined and preserved. An important manoeuvre is to divide the vestibular aqueduct so as to release the posterior fossa dura from the labyrinthine block-this manoeuvre does not lead to hearing loss.

Bone drilling should expose middle fossa dura, posterior fossa dura (Trautmann's triangle) (Fig. 3) and the superior petrosal sinus. If the jugular bulb is high, then this can be pushed down up by approximately $1 \mathrm{~cm}$.

In our experience, this should provide enough exposure for most pathologies and we do not routinely include a retrosigmoid craniotomy as part of our exposure. Bone, however, can be removed from over the sigmoid sinus allowing for further dural retraction.

A curved dural incision is performed over the middle and posterior fossae extending towards the sinodural angle which marks the position of the superior petrosal sinus. The sinus can then be ligated with haemoclips and divided. The third cranial nerve is identified with careful elevation of the medial edge of the tentorium. Dissection can then proceed medial to lateral in the knowledge that the nerve has been identified. Identification of the medial edge of the tentorium also ensures that the tentorial incision is made at the appropriate angle; if the cut is made lateral to medial without identifying the medial tentorial edge, it is possible that the angle of incision is too acute to the medial edge in an anterior direction.

Once the tentorium is completely incised, the operative field is fully exposed with minimal brain retraction. In the case of a medially based petrous apex meningioma, the trigeminal nerve will usually be evident on the lateral aspect of the tumour along with the third nerve anteriorly and the fourth nerve more posteriorly. The other cranial nerves should be identified and preserved. The origin of tumour on the posterior petrous bone, with respect to the position of the porus, will determine how cranial nerves might be displaced or encased by the tumour.

The basilar artery is often displaced medially by a meningioma. Dissection should proceed in such a way as to be able to identify the artery and its branches as early as possible.
Fig. 5 Small residuum left to preserve cranial nerve function. Mild new V3 paraesthesiae only
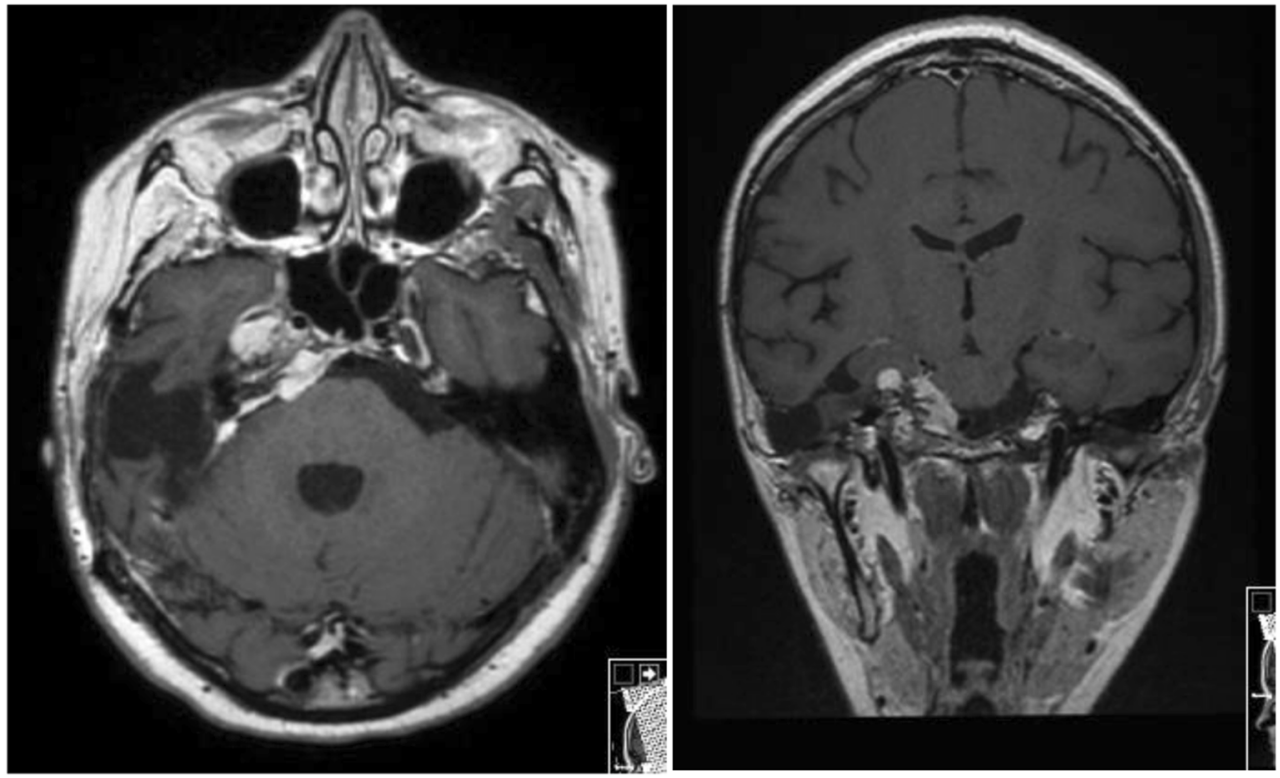
Meticulous closure can be difficult particularly around the superior petrosal sinus (Fig. 4). A small piece of fascia lata is used to cover the antrum and abdominal fat is harvested to fill the mastoid cavity defect. Fat in the antrum will compromise hearing.

\section{Complications}

Patients are exposed to the general risks of any neurosurgical procedure in terms of infection, including meningitis, bleeding, cerebrospinal fluid leak, fits, hemiparesis, thromboembolism and death.

Particular risks pertaining to this approach include cerebrospinal fluid rhinorrhoea, sub-cutaneous pseudomeningocoele, deafness, facial weakness and numbness, ophthalmoplegia, speech and swallowing difficulties, ataxia and temporal lobe damage including that which relates to disruption of the vein of Labbé and brainstem ischaemia as a result of posterior circulation vessel damage.

\section{Conclusion}

This is an important approach to master when faced with large petrous apex lesions, especially meningiomata. It is also useful in approaching the antero-lateral brainstem and the upper and mid basilar trunk.

The opening of both the supra- and infra-tentorial compartments gives unparalleled wide exposure of the pathology, improves chances of early identification and preservation of cranial nerves and other structures and avoids brain retraction (Fig. 5).
Open Access This article is distributed under the terms of the Creative Commons Attribution 4.0 International License (http:// creativecommons.org/licenses/by/4.0/), which permits unrestricted use, distribution, and reproduction in any medium, provided you give appropriate credit to the original author(s) and the source, provide a link to the Creative Commons license, and indicate if changes were made.

Publisher's Note Springer Nature remains neutral with regard to jurisdictional claims in published maps and institutional affiliations.

\section{References}

Papers of particular interest, published recently, have been highlighted as:

- Of importance

•• Of major importance

1. Grossi PM, Nonaka Y, Watanabe K, Fukushima T. The history of the combined supra- and infra-tentorial approach to the petroclival region. Neurosurg Focus. 2012;33(2):E8 This is an excellent summary of how the approach has developed over the years.

2. Morrison A, King TT. Experiences with a trans-labyrinthine transtentorial approach to the cerebellopontine angle. Technical note. J Neurosurg. 1973;38:382-90 This is a landmark paper detailing the expansion of the standard trans-labyrinthine approach.

3.• Spetzler RF, Daspit CP, Pappas CTE. The combined supra- and infratentorial approach for lesions of the petrous and clival regions: experience with 46 cases. J Neurosurg. 1992;76:588-99 Spetzler is one of the key architects of this approach and this paper summarises the surgical technique, which has changed little over the years. 